\title{
THE TIME CONSTANT AND CRITICAL PROBABIL- ITIES IN PERCOLATION MODELS
}

\author{
LEANDRO P. R. PIMENTEL ${ }^{1}$ \\ Institut de Mathématiques, École Polytechinique Fédérale de Lausanne, CH-1015 Lausanne, \\ Switzerland \\ email: leandro.pimentel@epfl.ch
}

Submitted 3 February 2005, accepted in final form 19 July 2006

AMS 2000 Subject classification: 60K35, 82D30

Keywords: Percolation, time constant, critical probabilities, Delaunay triangulations

\section{Abstract}

We consider a first-passage percolation (FPP) model on a Delaunay triangulation $\mathcal{D}$ of the plane. In this model each edge $\mathbf{e}$ of $\mathcal{D}$ is independently equipped with a nonnegative random variable $\tau_{\mathbf{e}}$, with distribution function $\mathbb{F}$, which is interpreted as the time it takes to traverse the edge. Vahidi-Asl and Wierman [9] have shown that, under a suitable moment condition on $\mathbb{F}$, the minimum time taken to reach a point $\mathbf{x}$ from the origin $\mathbf{0}$ is asymptotically $\mu(\mathbb{F})|\mathbf{x}|$, where $\mu(\mathbb{F})$ is a nonnegative finite constant. However the exact value of the time constant $\mu(\mathbb{F})$ still a fundamental problem in percolation theory. Here we prove that if $\mathbb{F}(0)<1-p_{c}^{*}$ then $\mu(\mathbb{F})>0$, where $p_{c}^{*}$ is a critical probability for bond percolation on the dual graph $\mathcal{D}^{*}$.

\section{Introduction}

First-passage percolation theory on periodic graphs was presented by Hammersley and Welsh [4] to model the spread of a fluid through a porous medium. In this paper we continue a study of planar first-passage percolation models on random graphs, initiated by Vahidi-Asl and Wierman [9], as follows. Let $\mathcal{P}$ denote the set of points realized in a two-dimensional homogeneous Poisson point process with intensity 1 . To each $\mathbf{v} \in \mathcal{P}$ corresponds an open polygonal region $\mathbf{C}_{\mathbf{v}}=\mathbf{C}_{\mathbf{v}}(\mathcal{P})$, the Voronoi tile at $\mathbf{v}$, consisting of the set of points of $\mathbb{R}^{2}$ which are closer to $\mathbf{v}$ than to any other $\mathbf{v}^{\prime} \in \mathcal{P}$. Given $\mathbf{x} \in \mathbb{R}^{2}$ we denote by $\mathbf{v}_{\mathbf{x}}$ the almost surely unique point in $\mathcal{P}$ such that $\mathbf{x} \in \mathbf{C}_{\mathbf{v}_{\mathbf{x}}}$. The collection $\left\{\mathbf{C}_{\mathbf{v}}: \mathbf{v} \in \mathcal{P}\right\}$ is called the Voronoi Tiling of the plane based on $\mathcal{P}$.

The Delaunay Triangulation $\mathcal{D}$ is the graph where the vertex set $\mathcal{D}_{v}$ equals $\mathcal{P}$ and the edge set $\mathcal{D}_{e}$ consists of non-oriented pairs $\left(\mathbf{v}, \mathbf{v}^{\prime}\right)$ such that $\mathbf{C}_{\mathbf{v}}$ and $\mathbf{C}_{\mathbf{v}^{\prime}}$ share a one-dimensional edge (Figure 1). One can see that almost surely each Voronoi tile is a convex and bounded polygon, and the graph $\mathcal{D}$ is a triangulation of the plane [7]. The Voronoi Tessellation $\mathcal{V}$ is the graph where the vertex set $\mathcal{V}_{v}$ is the set of vertices of the Voronoi tiles and the edge set $\mathcal{V}_{e}$ is the set

\footnotetext{
${ }^{1}$ RESEARCH SUPPORTED BY SWISS NATIONAL SCIENCE FOUNDATION GRANT 510767
} 


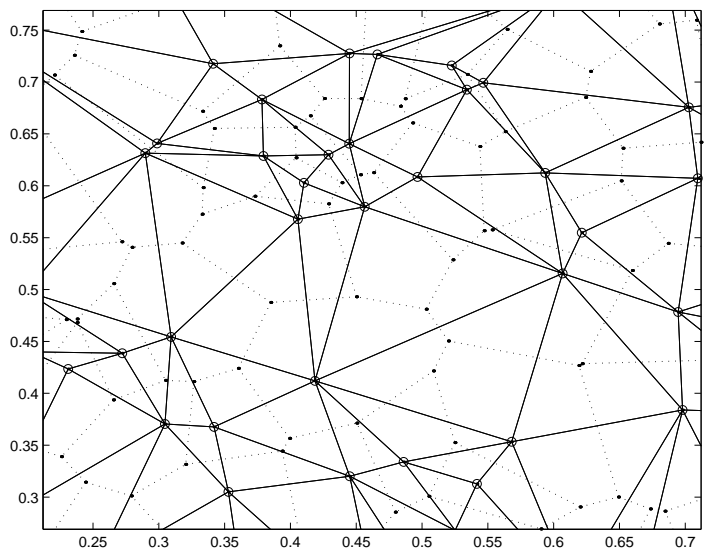

Figure 1: The Delaunay Triangulation and the Voronoi Tessellation.

of edges of the Voronoi tiles. The edges of $\mathcal{V}$ are segments of the perpendicular bisectors of the edges of $\mathcal{D}$. This establishes duality of $\mathcal{D}$ and $\mathcal{V}$ as planar graphs: $\mathcal{V}=\mathcal{D}^{*}$.

To each edge $\mathbf{e} \in \mathcal{D}_{e}$ is independently assigned a nonnegative random variable $\tau_{\mathbf{e}}$ from a common distribution $\mathbb{F}$, which is also independent of the Poisson point process that generates $\mathcal{P}$. From now on we denote $(\Omega, \mathcal{F}, \mathbb{P})$ the probability space induced by the Poisson point process $\mathcal{P}$ and the passage times $\left(\tau_{\mathbf{e}}\right)_{\mathbf{e} \in \mathcal{D}_{e}}$. The passage time $t(\gamma)$ of a path $\gamma$ in the Delaunay Triangulation is the sum of the passage times of the edges in $\gamma$. The first-passage time between two vertices $\mathbf{v}$ and $\mathbf{v}^{\prime}$ is defined by

$$
T\left(\mathbf{v}, \mathbf{v}^{\prime}\right):=\inf \left\{t(\gamma) ; \gamma \in \mathcal{C}\left(\mathbf{v}, \mathbf{v}^{\prime}\right)\right\},
$$

where $\mathcal{C}\left(\mathbf{v}, \mathbf{v}^{\prime}\right)$ the set of all paths connecting $\mathbf{v}$ to $\mathbf{v}^{\prime}$. Given $\mathbf{x}, \mathbf{y} \in \mathbb{R}^{2}$ we define $T(\mathbf{x}, \mathbf{y}):=$ $T\left(\mathbf{v}_{\mathbf{x}}, \mathbf{v}_{\mathbf{y}}\right)$.

To state the main result of this work we require some definitions involving a bond percolation model on the Voronoi Tessellation $\mathcal{V}$. Such a model is constructed by choosing each edge of $\mathcal{V}$ to be open independently with probability $p$. An open path is a path composed of open edges. We denote $\mathbb{P}_{p}^{*}$ the law induced by the Poisson point process and the random state (open or not) of an edge. Given a planar graph $\mathcal{G}$ and $\mathbf{A}, \mathbf{B} \subseteq \mathbb{R}^{2}$ we say that a self-avoiding path $\gamma=\left(\mathbf{v}_{1}, \ldots, \mathbf{v}_{k}\right)$ is a path connecting $\mathbf{A}$ to $\mathbf{B}$ if $\left[\mathbf{v}_{1}, \mathbf{v}_{2}\right] \cap \mathbf{A} \neq \emptyset$ and $\left[\mathbf{v}_{k-1}, \mathbf{v}_{k}\right] \cap \mathbf{B} \neq \emptyset$ $([\mathbf{x}, \mathbf{y}]$ denotes the line segment connecting $\mathbf{x}$ to $\mathbf{y})$. For $L>0$ let $A_{L}$ be the event that there exists an open path $\gamma=\left(\mathbf{v}_{j}\right)_{1 \leq j \leq h}$ in $\mathcal{V}$, connecting $\{0\} \times[0, L]$ to $\{3 L\} \times[0, L]$, and with $\mathbf{v}_{j} \in[0,3 L] \times[0, L]$ for all $j=2, \ldots, h-1$. In this case we also say that $\gamma$ crosses the rectangle $[0,3 L] \times[0, L]$. Define the function

$$
\eta^{*}(p):=\liminf _{L \rightarrow \infty} \mathbb{P}_{p}^{*}\left(A_{L}\right)
$$

and consider the percolation threshold,

$$
p_{c}^{*}:=\inf \left\{p>0: \eta^{*}(p)=1\right\} .
$$

We have that $p_{c}^{*} \in(0,1)$, which follows by standard arguments in percolation theory. For more in percolation thresholds on Voronoi tilings we refer to $[1,2,11]$. 
Theorem 1 If $\mathbb{F}(0)<1-p_{c}^{*}$ then there exist constants $c_{j}>0$ such that for all $n \geq 1$

$$
\mathbb{P}\left(T(\mathbf{0}, \mathbf{n})<c_{1} n\right) \leq c_{2} \exp \left(-c_{3} n\right),
$$

where $\mathbf{0}:=(0,0)$ and $\mathbf{n}:=(n, 0)$.

To show the importance of Theorem 1 we recall two fundamental results proved by Vahidi-Asl and Wierman $[9,10]$. Consider the growth process

$$
\mathbf{B}_{\mathbf{x}}(t):=\left\{\mathbf{y} \in \mathbb{R}^{2}: \mathbf{y} \in c\left(\mathbf{C}_{\mathbf{v}}\right) \text { with } \mathbf{v} \in \mathcal{D}_{v} \text { and } T\left(\mathbf{v}_{\mathbf{x}}, \mathbf{v}\right) \leq t\right\} .
$$

where $c(\mathbf{C})$ denotes the closure of $\mathbf{C} \in \mathbb{R}^{2}$. Set

$$
\mu(\mathbb{F}):=\inf _{n>0} \frac{\mathbb{E} T(\mathbf{0}, \mathbf{n})}{n} \in[0, \infty] .
$$

and let $\tau_{1}, \tau_{2}, \tau_{3}$ be independent random variables with distribution $\mathbb{F}$. If

$$
\mathbb{E}\left(\min _{j=1,2,3}\left\{\tau_{j}\right\}\right)<\infty
$$

then $\mu(\mathbb{F})<\infty$ and for all unit vectors $\overrightarrow{\mathbf{x}} \in S^{1}(|\overrightarrow{\mathbf{x}}|=1) \mathbb{P}$-a.s.

$$
\lim _{n \rightarrow \infty} \frac{T(\mathbf{0}, n \overrightarrow{\mathbf{x}})}{n}=\lim _{n \rightarrow \infty} \frac{\mathbb{E} T(\mathbf{0}, \mathbf{n})}{n}=\mu(\mathbb{F}) .
$$

Further, if

$$
\mathbb{E}\left(\min _{j=1,2,3}\left\{\tau_{j}\right\}^{2}\right)<\infty
$$

and $\mu(\mathbb{F})>0$ then for all $\epsilon>0 \mathbb{P}$-a.s. there exists $t_{0}>0$ such that for all $t>t_{0}$

$$
(1-\epsilon) t \mathbf{D}(1 / \mu) \subseteq \mathbf{B}_{\mathbf{0}}(t) \subseteq(1+\epsilon) t \mathbf{D}(1 / \mu),
$$

where $\mathbf{D}(r):=\left\{\mathbf{x} \in \mathbb{R}^{2}:|\mathbf{x}| \leq r\right\}$.

We note here that the asymptotic shape is an Euclidean ball due to the statistical invariance of the Poisson point process. Unfortunately the exact value of the time constant $\mu(\mathbb{F})$, as a functional of $\mathbb{F}$, still a basic problem in first-passage percolation theory. Our result provides a sufficient condition on $\mathbb{F}$ to ensure $\mu(\mathbb{F})>0$.

Corollary 1 Under assumption (3), if $\mathbb{F}(0)<1-p_{c}^{*}$ then $\mu(\mathbb{F}) \in(0, \infty)$.

Proof. Together with the Borel-Cantelli Lemma, Theorem 1 and (4) imply

$$
0<c_{1} \leq \liminf _{n \rightarrow \infty} \frac{T(\mathbf{0}, \mathbf{n})}{n}=\lim _{n \rightarrow \infty} \frac{T(\mathbf{0}, \mathbf{n})}{n}=\mu(\mathbb{F})<\infty,
$$

which is the desired result.

For FPP models on the $\mathbb{Z}^{2}$ lattice Kesten (1986) has shown that $\mathbb{F}(0)<1 / 2=p_{c}\left(\mathbb{Z}^{2}\right)$ (the critical probability for bond percolation on $\mathbb{Z}^{2}$ ) is a sufficient condition to get (2) by using a stronger version of the BK-inequality. Here we follow a different method and we apply a simple renormalization argument to obtain a similar result. We expect that our condition to get (2) is equivalent to

$$
\mathbb{F}(0)<p_{c}:=\inf \{p>0 ; \theta(p)=1\},
$$

where $\theta(p)$ is the probability that bond percolation on $\mathcal{D}$ occurs with density $p$, since it is conjectured that $p_{c}+p_{c}^{*}=1$ (duality) for many planar graphs. In fact, by combining Corollary 1 with (6) we have: 


\section{Corollary 2}

$$
1 \leq p_{c}+p_{c}^{*} .
$$

Proof. To see this assume we have a first-passage percolation model on $\mathcal{D}$ with

$$
\mathbb{P}\left(\tau_{\mathbf{e}}=0\right)=1-\mathbb{P}\left(\tau_{\mathbf{e}}=1\right)=\mathbb{F}(0)=1-p>p_{c}^{*} .
$$

Then $\mathbb{P}$-a.s. there exists an infinite cluster $\mathcal{W} \subseteq \mathcal{D}$ composed by edges e with $\tau_{\mathbf{e}}=0$. Denote by $T(\mathbf{0}, \mathcal{W})$ the first-passage time from $\mathbf{0}$ to $\mathcal{W}$. Then for all $t>T(\mathbf{0}, \mathcal{W})$ we have that $\mathbf{B}_{\mathbf{0}}(t)$ is an unbounded set. By (6) (since such a distribution satisfies (3) and (5)), this implies that $\mu(\mathbb{F})=\mu(p)=0$ if $1-p>p_{c}$. On the other hand, by Corollary $1, \mu(p)>0$ if $1-p<1-p_{c}^{*}$, and so (2) must hold.

Other passage times have been considered in the literature such as $T\left(\mathbf{0}, \mathbf{H}_{n}\right)$, where $\mathbf{H}_{n}$ is the hyperplane consisting of points $\mathbf{x}=\left(x^{1}, x^{2}\right)$ so that $x_{1}=n$, and $T\left(\mathbf{0}, \partial[-n, n]^{2}\right)$. The arguments in this article can be used to prove the analog of Theorem 1 when $T(\mathbf{0}, \mathbf{n})$ is replaced by $T\left(\mathbf{0}, \mathbf{H}_{n}\right)$ or $T\left(\mathbf{0}, \partial[-n, n]^{2}\right)$. For site versions of FPP models the method works as well if we change the condition on $\mathbb{F}$ to $\mathbb{F}(0)<1-\bar{p}_{c}$, where now $\bar{p}_{c}$ is the critical probability for site percolation. Similarly to Corollary 2, in this case one can also obtain the inequality $1 / 2 \leq \bar{p}_{c}$. For more details we refer to [8].

\section{Renormalization}

For the moment we assume that $\mathbb{F}$ is Bernoulli with parameter $p$. Let $L \geq 1$ be a parameter whose value will be specified later. Let $\mathbf{z}=\left(z^{1}, z^{2}\right) \in \mathbb{Z}^{2}$ and

$$
|\mathbf{z}|_{\infty}:=\max _{j=1,2}\left\{\left|z^{j}\right|\right\} .
$$

Denote $\mathbf{C}_{z}$ the circuit composed by sites $\mathbf{z}^{\prime} \in \mathbb{Z}^{2}$ with $\left|\mathbf{z}-\mathbf{z}^{\prime}\right|_{\infty}=2$. For each $\mathbf{A} \subseteq \mathbb{R}^{2}$, we denote by $\partial \mathbf{A}$ its boundary. For each $\mathbf{z} \in \mathbb{Z}^{2}$ and $r \in\{j / 2: j \in \mathbb{N}\}$ consider the box

$$
\mathbf{B}_{z}^{r L}:=L z+[-r L, r L]^{2} .
$$

Divide $\mathbf{B}_{\mathbf{z}}^{L / 2}$ into thirty-six sub-boxes with the same size and declare that $B_{\mathbf{z}}^{L / 2}$ is a full box if all these thirty-six sub-boxes contain at least one point of $\mathcal{P}$. Let

$$
H_{\mathbf{z}}^{L}:=\left[\mathbf{B}_{\mathbf{z}^{\prime}}^{L / 2} \text { is a full box } \forall \mathbf{z}^{\prime} \in \mathbf{C}_{\mathbf{z}}\right] .
$$

Let $\mathcal{C}_{L}$ be the set of all self-avoiding paths $\gamma=\left(\mathbf{v}_{j}\right)_{1 \leq j \leq h}$ in $\mathcal{D}$, connecting $\partial \mathbf{B}_{\mathbf{z}}^{L / 2}$ to $\partial \mathbf{B}_{\mathbf{z}}^{3 L / 2}$ and with $\mathbf{C}_{\mathbf{v}_{j}} \cap \mathbf{B}_{\mathbf{z}}^{3 L / 2}$ for all $j=2, \ldots, h-1$. Let

$$
G_{z}^{L}:=\left[t(\gamma) \geq 1 \forall \gamma \in \mathcal{C}_{L}\right] .
$$

We say that $\mathbf{B}_{\mathbf{z}}^{L / 2}$ is a good box (or that $\mathbf{z}$ is a good point) if

$$
Y_{\mathbf{z}}^{L}:=\mathbb{I}\left(H_{\mathbf{z}}^{L} \cap G_{\mathbf{z}}^{L}\right)=1,
$$

where $\mathbb{I}(E)$ denotes the indicator function of the event $E$. 


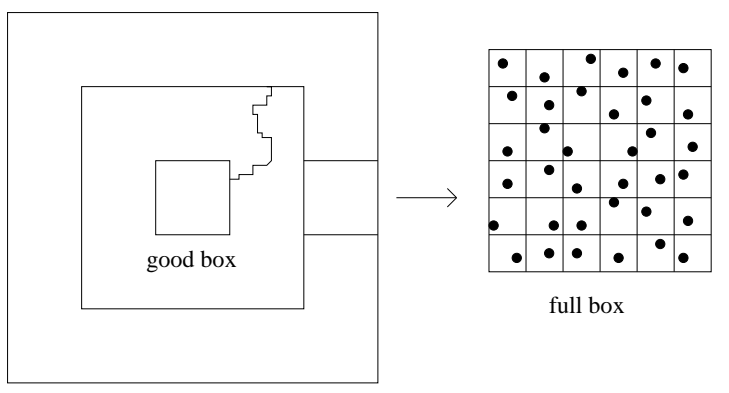

Figure 2: Renormalization

Lemma 1 If $\mathbb{P}\left(\tau_{\mathbf{e}}=0\right)=1-p<1-p_{c}^{*}$ then

$$
\lim _{L \rightarrow \infty} \mathbb{P}\left(Y_{\mathbf{0}}^{L}=1\right)=1 .
$$

Proof. First notice that

$$
\mathbb{P}\left(Y_{\mathbf{0}}^{L}=0\right) \leq \mathbb{P}\left(\left(H_{\mathbf{0}}^{L}\right)^{c}\right)+\mathbb{P}\left(\left(G_{\mathbf{0}}^{L}\right)^{c}\right) .
$$

By the definition of a two-dimensional homogeneous Poisson point process,

$$
\lim _{L \rightarrow \infty} \mathbb{P}\left(\left(H_{\mathbf{0}}^{L}\right)^{c}\right)=0
$$

Now, let $X_{\mathbf{e}^{*}}:=\tau_{\mathbf{e}}$, where $\mathbf{e}^{*}$ is the edge in $\mathcal{V}_{e}$ (the Voronoi tessellation) dual to e. Then $\left\{X_{\mathbf{e}^{*}} ; \mathbf{e}^{*} \in \mathcal{V}_{e}\right\}$ defines a bond percolation model on $\mathcal{V}$ with law $\mathbb{P}_{p}^{*}$. Consider the rectangles

$$
\begin{gathered}
R_{L}^{1}:=[L / 2,3 L / 2] \times[-3 L / 2,3 L / 2], R_{L}^{2}:=[-3 L / 2,3 L / 2] \times[L / 2,3 L / 2] \\
R_{L}^{3}:=[-3 L / 2,-L / 2] \times[-3 L / 2,3 L / 2] \text { and } R_{L}^{4}:=[-3 L / 2,3 L / 2] \times[-3 L / 2,-L / 2] .
\end{gathered}
$$

We denote by $A_{L}^{i}$ the event $A_{L}$ (recall the definition of $p_{c}^{*}$ ) but now translate to the rectangle $R_{L}^{i}$, and by $F_{L}$ the event that an open circuit $\sigma^{*}$ in $\mathcal{V}$ which surrounds $B_{\mathbf{0}}^{L / 2}$ and lies inside $B_{\mathbf{0}}^{3 L / 2}$ does not exist. Thus one can easily see that

$$
\cap_{i=1}^{4} A_{L}^{i} \subseteq\left(F_{L}\right)^{c}
$$

Notice that if there exists an open circuit $\sigma^{*}$ in $\mathcal{V}$ which surrounds $B_{\mathbf{0}}^{L / 2}$ and lies inside $B_{\mathbf{0}}^{3 L / 2}$, then every path $\gamma$ in $\mathcal{C}_{L}$ has an edge crossing with $\sigma^{*}$ and thus $t(\gamma) \geq 1$. Therefore,

$$
\mathbb{P}\left(\left(G_{0}^{L}\right)^{c}\right) \leq \mathbb{P}_{p}^{*}\left(F_{L}\right) \leq 4\left(1-\mathbb{P}_{p}^{*}\left(A_{L}\right)\right)
$$

Since $p>p_{c}^{*}$, by using (8), (9), (10) and the definition of $p_{c}^{*}$, we get Lemma 1 .

To obtain some sort of independence between the random variables $Y_{\mathbf{z}}^{L}$ we shall study some geometrical aspects of Voronoi tilings. Given $\mathbf{A} \subseteq \mathbb{R}^{2}$, let $\mathcal{I}_{\mathcal{P}}(\mathbf{A})$ be the sub-graph of $\mathcal{D}$ composed of vertices $\mathbf{v}_{1}$ in $\mathcal{D}_{v}$ and edges $\left(\mathbf{v}_{2}, \mathbf{v}_{3}\right)$ in $\mathcal{D}_{e}$ so that $\mathbf{C}_{\mathbf{v}_{i}} \cap \mathbf{A} \neq \emptyset$ for all $i=1,2,3$. 
Lemma 2 Let $L>0$ and $\mathbf{z} \in \mathbb{Z}^{2}$. Assume that $\mathcal{P}$ and $\mathcal{P}^{\prime}$ are two configurations of points so that $\mathcal{P} \cap \mathbf{B}_{\mathbf{z}}^{5 L / 2}=\mathcal{P}^{\prime} \cap \mathbf{B}_{\mathbf{z}}^{5 L / 2}$ and that $\mathbf{B}_{\mathbf{z}^{\prime}}^{L / 2}$ is a full box with respect to $\mathcal{P}$, for all $\mathbf{z}^{\prime} \in \mathbf{C}_{\mathbf{z}}$. Then $\mathcal{I}_{\mathcal{P}}\left(\mathbf{B}_{\mathbf{z}}^{3 L / 2}\right)=\mathcal{I}_{\mathcal{P}^{\prime}}\left(\mathbf{B}_{\mathbf{z}}^{3 L / 2}\right)$.

Proof. By the definition of the Delaunay Triangulation, Lemma 2 holds if we prove that

$$
\mathbf{C}_{\mathbf{v}}(\mathcal{P}) \cap \mathbf{B}_{\mathbf{z}}^{3 L / 2} \neq \emptyset \Rightarrow \mathbf{C}_{\mathbf{v}}(\mathcal{P})=\mathbf{C}_{\mathbf{v}}\left(\mathcal{P}^{\prime}\right)
$$

To prove this we claim that

$$
\mathbf{C}_{\mathbf{v}}(\mathcal{P}) \cap \mathbf{B}_{\mathbf{z}}^{3 L / 2} \neq \emptyset \Rightarrow \mathbf{C}_{\mathbf{v}}(\mathcal{P}) \subseteq \mathbf{B}_{\mathbf{z}}^{2 L} .
$$

If (12) does not hold then there exist $\mathbf{x}_{1} \in \partial \mathbf{B}_{\mathbf{z}}^{3 L / 2} \cap \mathbf{C}_{\mathbf{v}}(\mathcal{P})$ and $\mathbf{x}_{2} \in \partial \mathbf{B}_{\mathbf{z}}^{2 L} \cap \mathbf{C}_{\mathbf{v}}(\mathcal{P}$ ) (by convexity of Voronoi tilings). Since every box $B_{\mathbf{z}^{\prime}}^{L / 2}$ with $\left|\mathbf{z}-\mathbf{z}^{\prime}\right|_{\infty}=2$ is a full box, there exist $\mathbf{v}_{1}, \mathbf{v}_{2} \in \mathcal{P}$ so that

$$
\left|\mathbf{v}_{1}-\mathbf{x}_{1}\right| \leq \sqrt{2} L / 6 \text { and }\left|\mathbf{v}_{2}-\mathbf{x}_{2}\right| \leq \sqrt{2} L / 6
$$

Although, $\mathbf{x}_{1}$ and $\mathbf{x}_{2}$ belong to $\mathbf{C}_{\mathbf{v}}(\mathcal{P})$ and so

$$
\left|\mathbf{v}-\mathbf{x}_{1}\right| \leq\left|\mathbf{v}_{1}-\mathbf{x}_{1}\right| \text { and }\left|\mathbf{v}-\mathbf{x}_{2}\right| \leq\left|\mathbf{v}_{2}-\mathbf{x}_{2}\right| \text {. }
$$

Thus,

$$
L / 2 \leq\left|\mathbf{x}_{1}-\mathbf{x}_{2}\right| \leq\left|\mathbf{x}_{1}-\mathbf{v}\right|+\left|\mathbf{x}_{2}-\mathbf{v}\right| \leq \sqrt{2} L / 3,
$$

which leads to a contradiction since $\sqrt{2} / 3<1 / 2$. By an analogous argument, one can prove that

$$
\mathbf{C}_{\mathbf{v}^{\prime}}\left(\mathcal{P}^{\prime}\right) \cap\left(\mathbf{B}_{\mathbf{z}}^{5 L / 2}\right)^{c} \neq \emptyset \Rightarrow \mathbf{C}_{\mathbf{v}^{\prime}}\left(\mathcal{P}^{\prime}\right) \subseteq\left(\mathbf{B}_{\mathbf{z}}^{2 L}\right)^{c} .
$$

Now suppose (11) does not hold. Without lost of generality, we may assume that there exists $\mathbf{v} \in \mathcal{P}$ with $\mathbf{C}_{\mathbf{v}}(\mathcal{P}) \cap \mathbf{B}_{\mathbf{z}}^{3 L / 2} \neq \emptyset$ and $\mathbf{x} \in \mathbf{C}_{\mathbf{v}}(\mathcal{P})$ with $\mathbf{x} \notin \mathbf{C}_{\mathbf{v}}\left(\mathcal{P}^{\prime}\right)$. So $\mathbf{x} \in \mathbf{C}_{\mathbf{v}^{\prime}}\left(\mathcal{P}^{\prime}\right)$ for some $\mathbf{v}^{\prime} \in \mathcal{P}^{\prime}$. Although, $\mathcal{P} \cap \mathbf{B}_{\mathbf{z}}^{5 L / 2}=\mathcal{P}^{\prime} \cap \mathbf{B}_{\mathbf{z}}^{5 L / 2}$ and then $\mathbf{v}^{\prime} \in\left(\mathbf{B}_{\mathbf{z}}^{5 L / 2}\right)^{c}$, which is a contradiction with (12) and (13).

For each $l \geq 1$, we say that the collection of random variables $\left\{Y_{\mathbf{z}}: \mathbf{z} \in \mathbb{Z}^{2}\right\}$ is $l$-dependent if $\left\{Y_{\mathbf{z}}: \mathbf{z} \in \mathbf{A}\right\}$ and $\left\{Y_{\mathbf{z}}: \mathbf{z} \in \mathbf{B}\right\}$ are independent whenever

$$
l<d_{\infty}(\mathbf{A}, \mathbf{B}):=\min \left\{\left|\mathbf{z}-\mathbf{z}^{\prime}\right|_{\infty}: \mathbf{z} \in \mathbf{A} \text { and } \mathbf{z}^{\prime} \in \mathbf{B}\right\} .
$$

Combining Lemma 2 with the translation invariance and the independence property of the Poisson point process we obtain:

Lemma 3 For all $L>0,\left\{Y_{\mathbf{z}}^{L}: \mathbf{z} \in \mathbb{Z}^{2}\right\}$ is a 5-dependent collection of identically distributed Bernoulli random variables.

Denote $Y^{L}:=\left\{Y_{\mathbf{z}}^{L} ; \mathbf{z} \in \mathbb{Z}^{2}\right\}$ and let $M_{m}\left(Y^{L}\right)$ be the maximum number of pairwise disjoint good circuits in $\mathbb{Z}^{2}$, surrounding the origin and lying inside the box $[-m, m]^{2}$.

Lemma 4 If $\mathbb{F}(0)<1-p_{c}^{*}$ then there exists $L_{0}>0$ and $c_{j}=c_{j}\left(L_{0}\right)>0$ such that

$$
\mathbb{P}\left(M_{m}\left(Y^{L_{0}}\right) \leq c_{1} m\right) \leq \exp \left(-c_{2} m\right)
$$


Proof. Combining Lemmas 1 and 3 with and Theorem 0.0 of Ligget, Schonman and Stacey [6], one gets that $Y^{L}$ is dominated from below by a collection $X^{L}:=\left\{X_{z}^{L} ; z \in \mathbb{Z}^{2}\right\}$ of i.i.d. Bernoulli random variables with parameter $\rho(L) \rightarrow 1$ when $L \rightarrow \infty$. But for $\rho_{L}$ sufficiently close to 1 , we can chose $c>0$ sufficiently small, so that the probability of the event that $M_{m}\left(X^{L}\right)<c m$ decays exponentially fast with $m$ (see Chapter 3 of Grimmett [3]). Together with domination, this proves Lemma 4.

The connection between the variable $M_{m}\left(Y^{L}\right)$ and the first-passage time $T(\mathbf{0}, \mathbf{n})$ is summarize by the following:

\section{Lemma 5}

$$
\frac{M_{n L^{-1}}^{L}}{6} \leq T(\mathbf{0}, \mathbf{n})
$$

Proof. We say that $\left(B_{\mathbf{z}_{j}}^{L / 2}\right)_{1 \leq j \leq h}$ is a circuit of good boxes if $\left(\mathbf{z}_{j}\right)_{1 \leq j \leq h}$ is a good circuit in $\mathbb{Z}^{2}$, and that $\left(B_{\mathbf{z}_{j}}^{L / 2}\right)_{1 \leq j \leq h}$ and $\left(B_{\mathbf{z}_{j}^{\prime}}^{L / 2}\right)_{1 \leq j \leq h^{\prime}}$ are $l$-distant if

$$
d_{\infty}\left(\left(\mathbf{z}_{j}\right)_{1 \leq j \leq k},\left(\mathbf{z}_{j}^{\prime}\right)_{1 \leq j \leq h^{\prime}}\right)>l .
$$

Denote $M_{m}^{L}:=M_{m}\left(Y^{L}\right)$. Notice that there exist at least $\left(M_{n L^{-1}}^{L} / 6\right)$ pairwise 5-distant circuits of good boxes surrounding the origin and lying inside $[-n, n]^{2} \subseteq \mathbb{R}^{2}$. Therefore, every path $\gamma$ between the origin and any point outside $[-n, n]^{2}$ must cross at least $\left(M_{n L^{-1}}^{L} / 6\right) 5$-distant circuits of good boxes. We claim this yields

$$
\frac{M_{n L^{-1}}^{L}}{6} \leq t(\gamma)
$$

Indeed, assume we take two 5-distant good boxes, say $\mathbf{B}_{\mathbf{z}_{1}}^{L / 2}$ and $\mathbf{B}_{\mathbf{z}_{2}}^{L / 2}$, connected by a path $\gamma$ in $\mathcal{D}$. Then $\gamma$ must contain two sub-paths in $\mathcal{D}$, say $\bar{\gamma}_{i}=\left(\mathbf{v}_{j}^{i}\right)_{1 \leq j \leq h_{i}}$ for $i=1,2$, connecting $\partial \mathbf{B}_{\mathbf{z}_{i}}^{3 L / 2}$ to $\partial \mathbf{B}_{\mathbf{z}_{i}}^{5 L / 2}$ and with $\mathbf{C}_{\mathbf{v}_{j}^{i}} \cap \mathbf{B}_{\mathbf{z}_{i}}^{3 L / 2}$ for all $j=2, \ldots, h_{i}-1$. Since $B_{\mathbf{z}_{1}}^{L / 2}$ and $B_{\mathbf{z}_{2}}^{L / 2}$ are 5 -distant good boxes, by Lemma 2, these sub-paths must be edge disjoint. By the definition of a good box, $t\left(\bar{\gamma}_{1}\right) \geq 1$ and $t\left(\bar{\gamma}_{2}\right) \geq 1$, which yields

$$
2 \leq t\left(\bar{\gamma}_{1}\right)+t\left(\bar{\gamma}_{2}\right) \leq t(\gamma)
$$

By repeating this argument inductively (on the number of good boxes which are crossed by $\gamma$ ) one can get (14). Lemma 5 follows directly from (14).

Now we are ready to prove Theorem 1.

Proof. Together with Lemma 5, Lemma 4 implies Theorem 1 under (7). For the general case, assume $\mathbb{F}(0)=\mathbb{P}\left(\tau_{\mathbf{e}}=0\right)<1-p_{1}$. Fix $\epsilon>0$ so that $\mathbb{F}(\epsilon)<1-p_{c}^{*}$ (we can do so since $\mathbb{F}$ is right-continuous). Define the auxiliary process $\tau_{\mathbf{e}}^{\epsilon}:=\mathbb{I}\left(\tau_{\mathbf{e}}>\epsilon\right)$ and denote by $T^{\epsilon}$ the first-passage time associated to the collection $\left\{\tau_{\mathbf{e}}^{\epsilon}: \mathbf{e} \in \mathcal{D}_{e}\right\}$. Thus $T^{\epsilon}(\mathbf{0}, \mathbf{n}) \leq \epsilon^{-1} T(\mathbf{0}, \mathbf{n})$. Since $\tau_{\mathbf{e}}^{\epsilon}$ has a Bernoulli distribution with parameter $\mathbb{P}\left(\tau_{\mathbf{e}}^{\epsilon}=0\right)=\mathbb{F}(\epsilon)<1-p_{c}^{*}$, together with the previous case this yields Theorem 1 .

\section{Acknowledgment}

This work was develop during my doctoral studies at Impa and I would like to thank my adviser, Prof. Vladas Sidoravicius, for his dedication and encouragement during this period. I also thank the whole administrative staff of IMPA for their assistance and CNPQ for financing my doctoral studies, without which this work would have not been possible. 


\section{References}

[1] B. Bollobas and O. Riordan. The critical probability for random Voronoi percolation in the plane is 1/2. Preprint available from arXiv.org:math/0410336.

[2] B. Bollobas and O. Riordan. Sharp thresholds and percolation in the plane. Preprint available from arXiv.org:math/0412510.

[3] G. Grimmett. Percolation (second edition). Springer (1999).

[4] J.M. Hammersley, D.J.A. Welsh. First-passage percolation, sub-additive process, stochastic network and generalized renewal theory. Springer-Verlag (1965), 61-110.

[5] H. Kesten. Aspects of first-passage percolation. Lectures Notes in Math. 1180, SpringerVerlag (1986), 125-264.

[6] T.M. Ligget, R.H. Schonmann and A.M. Stacey. Domination by product measures. Ann. Probab. 25 (1997), 71-95.

[7] J. Moller. Lectures on random Voronoi tessellations. Lectures Notes in Stat. 87, SpringerVerlag (1991).

[8] L.P.R. Pimentel. Competing growth, interfaces and geodesics in first-passage percolation on Voronoi tilings. Phd Thesis, IMPA, Rio de Janeiro (2004).

[9] M.Q. Vahidi-Asl and J.C. Wierman. First-passage percolation on the Voronoi tessellation and Delaunay triangulation. Random Graphs 87 (M. Karonske, J. Jaworski and A. Rucinski, eds.) Wiley (1990), 341-359.

[10] M.C. Vahidi-Asl and J.C. Wierman. A shape result for first-passage percolation on the Voronoi tessellation and Delaunay triangulation. Random Graphs 89 (A. Frieze and T. Luczak, eds.), Wiley (1992), 247-262.

[11] A. Zvavitch. The critical probability for Voronoi percolation. MSc. thesis, Weizmann Institute of Science (1996). 